\title{
Water dynamics in the hydration shells of biological and non-biological polymers
}

Cite as: J. Chem. Phys. 150, 234904 (2019); https://doi.org/10.1063/1.5096392

Submitted: 17 March 2019 . Accepted: 29 May 2019 . Published Online: 21 June 2019

Silvina Cerveny (iD), and Jan Swenson (iD)

\section{COLLECTIONS}

Paper published as part of the special topic on Chemical Physics of Supercooled Water Note: This paper is part of a JCP Special Topic on Chemical Physics of Supercooled Water.
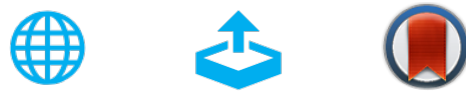

View Online

\section{ARTICLES YOU MAY BE INTERESTED IN}

Is water one liquid or two?

The Journal of Chemical Physics 150, 234503 (2019); https://doi.org/10.1063/1.5096460

Water in the hydrated protein powders: Dynamic and structure

The Journal of Chemical Physics 150, 204504 (2019); https://doi.org/10.1063/1.5096881

Motions of water and solutes-Slaving versus plasticization phenomena

The Journal of Chemical Physics 150, 124902 (2019); https://doi.org/10.1063/1.5030064

\section{Lock-in Amplifiers up to $600 \mathrm{MHz}$}
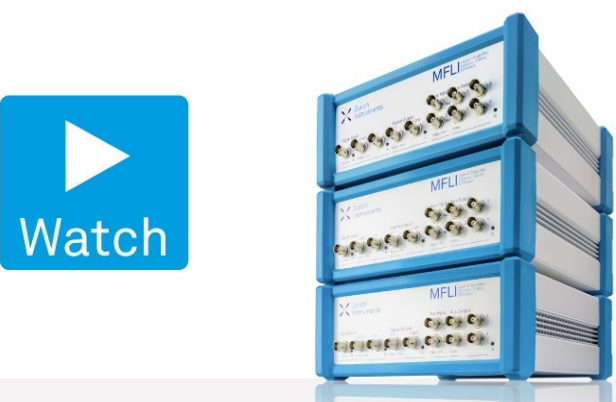

J. Chem. Phys. 150, 234904 (2019); https://doi.org/10.1063/1.5096392

150,234904 (c) 2019 Author(s). 


\title{
Water dynamics in the hydration shells of biological and non-biological polymers
}

\author{
Cite as: J. Chem. Phys. 150, 234904 (2019); doi: 10.1063/1.5096392 \\ Submitted: 17 March 2019 - Accepted: 29 May 2019 • \\ Published Online: 21 June 2019
}

Silvina Cerveny ${ }^{1,2, a)}$ (D) and Jan Swenson ${ }^{3}$

\begin{abstract}
AFFILIATIONS
${ }^{1}$ Centro de Física de Materiales (CSIC-UPV/EHU)-Material Physics Centre (MPC), Paseo Manuel de Lardizabal 5 , 20018 San Sebastián, Spain

${ }^{2}$ Donostia International Physics Center, Paseo Manuel de Lardizabal 4, 20018 San Sebastián, Spain

${ }^{3}$ Department of Physics, Chalmers University of Technology, SE-412 96 Göteborg, Sweden
\end{abstract}

Note: This paper is part of a JCP Special Topic on Chemical Physics of Supercooled Water.

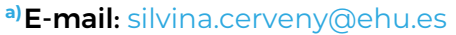

\begin{abstract}
The dynamics of water at supercooled temperatures in aqueous solutions of different types of solutes has been deeply analyzed in the literature. In these previous works and in most of the cases, a single relaxation of water molecules is observed. In this work, we analyze the dynamics of water in solutions for which a dual relaxation of water molecules is experimentally measured. We discuss the criteria for observing these two water relaxations in these specific solutions and their most likely origins. We also discuss how these two water relaxations relate to the relaxation behavior of bulk water and how the slower one is coupled to the solute dynamics and is essential for the dynamics and functional properties of proteins.
\end{abstract}

Published under license by AIP Publishing. https://doi.org/10.1063/1.5096392

\section{INTRODUCTION}

A solvation shell is the liquid at the interface of any chemical compound (biological or nonbiological) in a solution. When the liquid is water, the solvation shell is called hydration shell, which for proteins and DNA becomes very relevant because it gives them the flexibility to perform their catalytic job and screens and mediates interactions between biomolecules favoring conformational changes and creating preferred diffusive routes. For nonbiopolymers, the solvation shell is also of a great interest as it has implications in technological advances of practical interests. In both cases, the interfacial region exhibits distinct properties from the bulk aqueous phase because it is normally greatly affected by the solute itself.

For the particular case of proteins in our body, the interfacial region comprises between one or two layers of water molecules (whereas in well-diluted solutions, this interfacial region extends up to 6 layers $\left.^{1}\right)$. Beyond these layers $(\sim 6-20 \AA)$, bulklike water is also found. For protein solutions at physiological temperatures, it is possible to distinguish two different water relaxations. For instance, femtosecond fluorescence experiments ${ }^{2}$ detected two water relaxations in solutions of myoglobin (one slower at $\sim 20-200$ ps and one faster at 1-8 ps), which represent the local and the collective network restructuring of water, respectively. Close to room temperature, other methods such as $2 \mathrm{D}-\mathrm{IR}^{3}$ or fluorescence Stokes shift ${ }^{4}$ have independently detected the faster or the slower water relaxation. Molecular dynamics simulations have also analyzed the hydration water of proteins. In this case, water close to the protein also exhibits two different relaxations. ${ }^{5-7}$ At lower temperatures (in the supercooled region of water), it is easier to discriminate between different relaxations since rotational and translational motions become decoupled. Experiments by nuclear magnetic resonance (NMR) and dielectric spectroscopy, which cover very broad frequency and temperature ranges, also revealed two water relaxations in solutions of proteins. Previous studies showed that in hydrated lysozyme, ${ }^{8,9}$ bovine serum albumin at sufficiently high ${ }^{10}$ and low ${ }^{11}$ water concentrations, collagen and elastin, ${ }^{12}$ and even in short peptides of $n$-lysine, ${ }^{13}$ water exhibits two relaxations. In contrast to this behavior, most of the nonbiological aqueous solutions previously analyzed using the same experimental techniques showed only a single water relaxation. ${ }^{14}$ Therefore, the dual 
relaxation of water molecules seems to be exclusively related to solutions of biosystems (such as proteins or DNA).

Very recently, we extended these observations ${ }^{15}$ (i.e., the presence of two water relaxations) only previously seen in biological solutions to some specific nonbiological solutes. In particular, we have analyzed the synthetic rigid polymer poly(vinyl pyrrolidone) (PVP) of different molecular weights, $\varepsilon$-poly(lysine), a polymer made up of 25-35 residues of the single amino acid L-lysine ( $\varepsilon$-PLL), and dextran (a sugar). For these nonbiological solutions, we also have observed two water relaxations of similar characteristics to those previously seen in protein solutions. This finding implies that the occurrence of two independent water relaxations is not exclusively related to biopolymers and therefore neither related to the $3 \mathrm{D}$ conformation of biomolecules. The fact that water molecules in solutions can relax via two different processes is therefore a phenomenon more general than previously thought.

In this work, we will discuss results mainly obtained from differential scanning calorimetry (DSC) and dielectric spectroscopy (broadband dielectric spectroscopy, BDS) in solutions of nonbiological systems that show dynamics analogous to protein solutions. We will analyze solutions of different water contents and different $\mathrm{pH}$ values. Specifically, we want to address the origin of the slow water relaxation in these particular nonbiological solutes and why this relaxation is not observed in other water solutions or in water under strong confinements. We then discuss the implications of these conclusions for protein solutions, and finally, we will analyze the implications of these findings on the behavior of supercooled bulk water.

\section{EXPERIMENTAL DETAILS}

\section{A. Samples}

25 wt. \% $\varepsilon$-polylysine aqueous solution ( $\varepsilon$-PLL, average molecular weight $\mathrm{M}_{\mathrm{w}}=4.700 \mathrm{~g} / \mathrm{mol}$ and polydispersity index $\mathrm{M}_{\mathrm{w}} / \mathrm{M}_{\mathrm{n}}$ $=1.15$ ) was supplied by JNC Corporation (Japan). Poly(vinyl pyrrolidone) (PVP, $\mathrm{M}_{\mathrm{w}}=10.000,55.000$, and $160.000 \mathrm{~g} / \mathrm{mol}$ ), trilysine (3-Lys, $\left.\mathrm{M}_{\mathrm{w}}=402.5 \mathrm{~g} / \mathrm{mol}\right)$, and dextran $\left(\mathrm{M}_{\mathrm{w}}=70.000 \mathrm{~g} / \mathrm{mol}\right)$ were purchased from Aldrich Chemical. All the dry solutes (PVP, 3-Lys, $\varepsilon$-PLL, and dextran) were purified using an ion transfer resin (AG 501-X8, Bio-Rad Laboratories). To prepare the aqueous solutions, water was added to the appropriate concentration $\left(c_{w}\right)$ between 30 and $40 \mathrm{wt}$. \%. The mixtures were sealed for at least three months in order to achieve a good water distribution. In addition, we also prepared $\varepsilon$-PLL solutions with lower $\mathrm{pH}$ values $(\mathrm{pH}=7,8$, and 9) by adding $\mathrm{HCl}$ (Merk) without modification of the total water content (40 wt. \%).

\section{B. Structural characterization}

Differential scanning calorimeter (DSC) measurements were carried out using Q2000 TA equipment in the standard mode with cooling and heating rates of $10 \mathrm{~K} / \mathrm{min}$. $\varepsilon$-PLL, PVP, and dextran, in the dry state, are polymers that can be melted without any degradation, and therefore, the glass transition temperature of completely amorphous segments (without any secondary structure) can be determined through DSC. Fourier transform infrared (FTIR) spectroscopy was used to analyze the secondary structure of $\varepsilon$-PLL aqueous solutions. Different from other peptides, ${ }^{16,17}$ melted $\varepsilon$-PLL does not retain the $\beta$-sheet conformation observed in aqueous solutions (see Fig. S2 of the supplementary material). Infrared spectra were recorded at room temperature using a Jasco 6500 equipped with an attenuated total reflectance (ATR) unit. Small Angle X-ray Scattering (SAXS) was performed on the BL11 beam line of the ALBA Synchrotron facility, using an incident beam energy of $10 \mathrm{keV}$. The distance between the detector and the sample was $6.2 \mathrm{~m}$. This allowed us to observe the low $q$-range (down to $0.1 \mathrm{~nm}^{-1}$ ) corresponding to a real space length scale, $d$, of about $60 \mathrm{~nm}(d=2 \pi / q)$.

\section{Dynamical characterization}

The dynamics of the solutions was studied by broadband dielectric spectroscopy (BDS). To measure the complex dielectric permittivity in the frequency domain, $\varepsilon^{*}(\omega)=\varepsilon^{\prime}(\omega)-i \varepsilon^{\prime \prime}(\omega)$, we used a Novocontrol Alpha-S analyzer in the frequency range from $10^{-2}$ to $10^{6} \mathrm{~Hz}$ and an Agilent RF impedance analyzer 4192B over the frequency range of $10^{6}-10^{9} \mathrm{~Hz}$. Isothermal frequency scans were performed every $5 \mathrm{~K}$ in a temperature range from 140 to $170 \mathrm{~K}$ and every $2.5 \mathrm{~K}$ in the temperature range from 170 to $300 \mathrm{~K}$ with a temperature stability better than $0.1 \mathrm{~K}$. The samples were prepared forming a parallel-plate capacitor between gold-plated electrodes with a diameter of 10 and $20 \mathrm{~mm}$ for the high- and low-frequency ranges, respectively. In the gigahertz region $(0.2-20 \mathrm{GHz})$, a dielectric probe kit Hewlett-Packard (HP) HP-85070E with an open ended coaxial probe connected to a vector analyzer (VNA) HP-8361 was used. VNA was calibrated using air, water, and short circuit as calibration standards. In this case, $\varepsilon$-poly(lysine) solutions were prepared in a glass cylindrical container with a diameter of $20 \mathrm{~mm}$. The thickness of each sample was about $2 \mathrm{~cm}$. Measurements were performed from $275 \mathrm{~K}$ to $315 \mathrm{~K}$ in steps of $5 \mathrm{~K}$.

\section{RESULTS}

\section{A. Dynamics of $\varepsilon$-PLL aqueous solutions}

In a previous work, ${ }^{13}$ we analyzed the dynamics of $\varepsilon$-PLL at a water concentration of $40 \mathrm{wt}$. \% in broad temperature and frequency ranges without ice formation at any temperature. $\varepsilon$-PLL has no specific biological function as proteins have. However, in aqueous solutions, $\varepsilon$-PLL folds into a three-dimensional $\beta$-sheet structure. ${ }^{18,19}$ The temperature dependences of the relaxation processes observed in this solution are shown in Fig. 1(a). Broadening and relaxation strengths obtained from the fitting of the dielectric spectra are shown in Fig. S1 of the supplementary material, including a short discussion on their water concentration dependences. Following the results in Fig. 1, we detected three main relaxations: ${ }^{13}$ the two fastest relaxations correspond to the relaxation of water molecules, and the slowest relaxation is related to the $\alpha$-relaxation of the solute since it reaches a time scale of $100 \mathrm{~s}$ at $T_{g}$ measured by DSC. ${ }^{13}$ The same behavior (although at different time scales) is observed for solutions of nonbiological PVP and dextran, which do not have any specific conformation. At room temperature, the slow water relaxation is slower than bulk water, whereas the extrapolation of the fast water process [see the dashed line in Fig. 1(a)] seems to fit with the relaxation times of bulk water at higher temperatures. However, we cannot observe this fast relaxation at room temperature because it is hidden by the slow water process. In fact, Figs. 1(b) and 1(c) compare the real and the imaginary part of the complex permittivity 

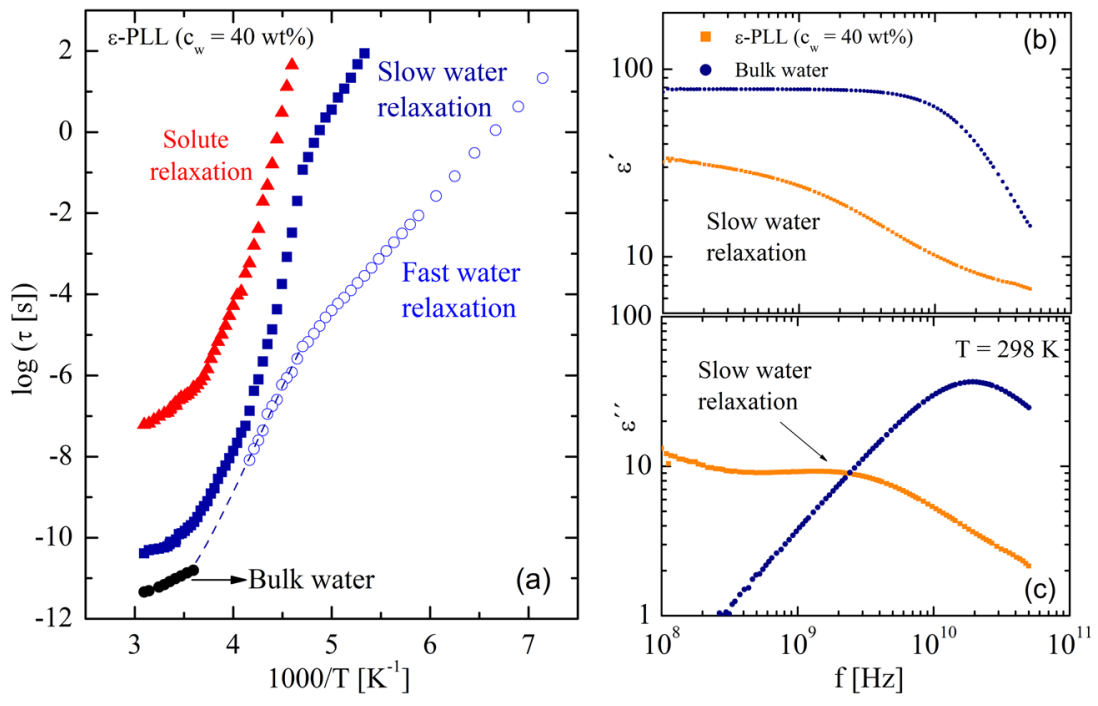

FIG. 1. (a) Temperature dependences of relaxation times, $\tau$, obtained by dielectric spectroscopy of $\varepsilon$-PLL $\left(c_{w}=40\right.$ wt. \%) and bulk water. [(b) and (c)] Comparison of the dielectric permittivity of bulk water and $\varepsilon-P L L$ at $T=298 \mathrm{~K}$. $\left(\varepsilon^{\prime}\right.$ and $\left.\varepsilon^{\prime \prime}\right)$ of bulk water and the slow water relaxation of $\varepsilon$-PLL. The slow water relaxation is much more extended in frequency than bulk water, revealing the higher heterogeneity degree of this water component in $\varepsilon$-PLL. Thus, the slow water relaxation with heterogeneous dynamics can be identified with the interfacial water in $\varepsilon$-PLL, but it also seems to involve surface groups of the solute molecules, as further discussed below.

More information about the origin of these relaxations can be obtained by analyzing different water contents in Fig. 2. The time scale of the fast water relaxation at these high water contents (where water-water interactions dominate) is almost independent of the water content, and therefore, it can be related to the relaxation of water molecules, which have not much interaction with

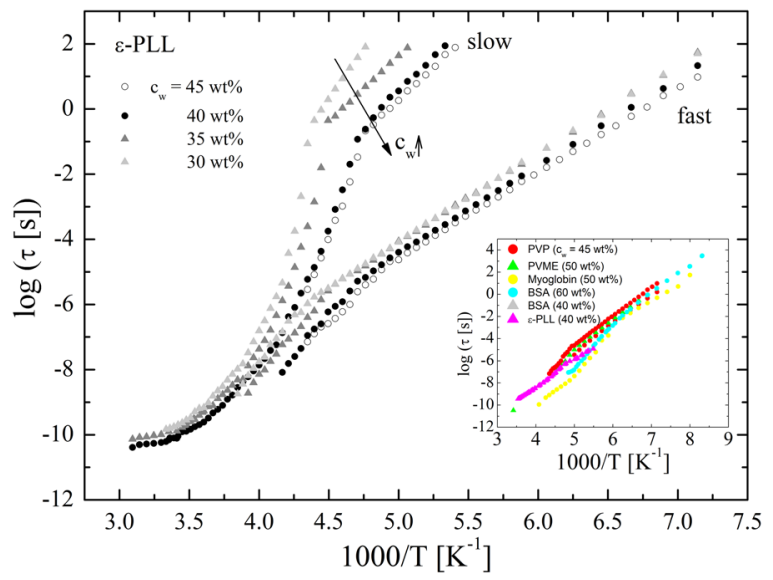

FIG. 2. Concentration dependences of the water relaxation times, $\tau$, obtained by dielectric spectroscopy of aqueous $\varepsilon-P L L$ solutions. The inset shows the fast water relaxation in other systems, as given in the figure, to evident its universality. solute molecules. Moreover, its activation energy $(\sim 0.5 \mathrm{eV})$ agrees with the universal activation energy for several aqueous solutions $\left(E_{a}=0.45-0.55 \mathrm{eV}\right)$ as well as for water molecules in other types of confinements, which implies that it can be attributed to a local relaxation of water molecules. In the inset of Fig. 2, the time scale of this water relaxation is compared with that observed in different protein solutions (BSA, ${ }^{20}$ myoglobin, ${ }^{21}$ and elastin ${ }^{22}$ ) at similar water contents. Since this water relaxation in $\varepsilon$-PLL and protein solutions shares a similar time scale and activation energy below $T_{g}$, we consider it to be of the same nature in all these systems.

Contrary to the fast water relaxation, the time scale of the slow water process depends on $c_{w}$ both below and above $T_{g}$. As its time scale is concentration dependent, the solute molecules should have substantial influence on this relaxation. The concentration dependence of the relaxation time is more evident at low temperatures (close to $T_{g}$ ), whereas at higher temperatures (well above $T_{g}$ ), the time scale of the relaxation becomes very similar for all the water contents. At $T>T_{g}$, the temperature dependence of its relaxation times follows a Vogel-Fulcher-Tammann (VFT) behavior, whereas at $T<T_{g}$, it becomes Arrhenius. The VFT behavior extends to $0.1 \mathrm{~s}$ $(\log \tau=-1)$, i.e., it does not reach $100 \mathrm{~s}$ at $T_{g}$, indicating that this relaxation is not related to the glass transition observed by DSC. Therefore, it is not identified as the structural $\alpha$-relaxation of the solution but rather due to a faster $\alpha$-like relaxation of the hydration water, and associated surface groups of the solutes, as mentioned above and further discussed below.

\section{B. Dynamics of water in $\varepsilon$-PLL solutions at different $\mathrm{pH}$ values}

To analyze the influence of the conformation of $\varepsilon$-PLL on the water relaxations, we changed the $\mathrm{pH}$ of the solution. However, at lower $\mathrm{pH}$ values (7 and 8), water crystallizes (on both cooling and heating), and therefore, the dielectric measurements only cover the temperature interval from 120 to $200 \mathrm{~K}$. In this case, we therefore focus only on the behavior of the fast water relaxation. 

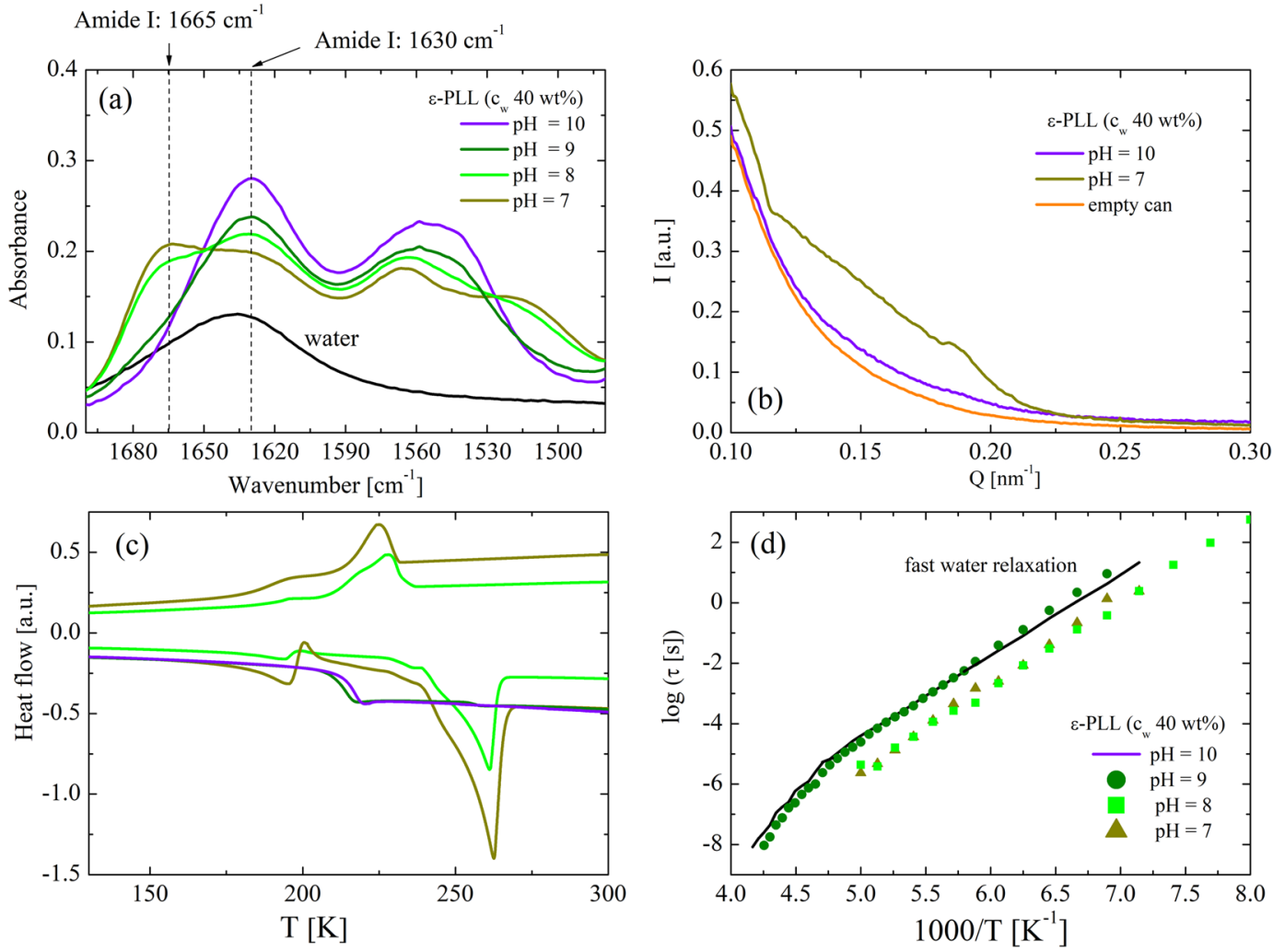

FIG. 3. (a) FTIR spectra for $\varepsilon$-PLL-water mixtures at $c_{w}=40$ wt. \% and different pH values. A spectrum of pure water is also shown for comparison. (b) SAXS data of $\varepsilon$-PLL $\left(c_{w}=40\right.$ wt. $\left.\%\right)$ at $\mathrm{pH}=10$ and 7. The empty can is also included. (c) Calorimetric data of the same solutions as in (a). Samples at pH $=8$ and 7 show crystallization. (d) Relaxation times of the fast water relaxation at different $\mathrm{pH}$ values.

Figure 3 shows the FTIR spectrum for different $\mathrm{pH}$ values. The amide I band, visible in the region of $1700-1600 \mathrm{~cm}^{-1}$, is used to determine the conformation of the peptide. ${ }^{23,24}$ For samples at $\mathrm{pH}=10$, the amide I band is located at $1630 \mathrm{~cm}^{-1}$ [see dashed lines in Fig. 3(a)], which indicates a $\beta$-sheet conformation. As the $\mathrm{pH}$ value decreases, a new band appears at $1665 \mathrm{~cm}^{-1}$ in addition to the $1630 \mathrm{~cm}^{-1}$ band, indicating that the conformation is a mix between $\beta$-sheets and $\beta$-turns, in agreement with previous studies. ${ }^{18,25,26}$ Figure 3 (b) shows the SAXS patterns in the $Q$ range of $0.1-0.3 \mathrm{~nm}^{-1}$ for the same $\varepsilon$-PLL solutions at $\mathrm{pH}=7$ and 10 and for the empty can. The main aim of this measurement was to analyze the differences between the amorphous sample $(\mathrm{pH}=10)$ and the sample that exhibits crystallization on cooling $(\mathrm{pH}=7)$, both having the same water content. It should be noted that most of the small-angle scattering observed in Fig. 3(b) is coming from the sample container as evidenced by the fact that the empty capillary shows a similar intensity increase toward low $Q$. At $\mathrm{pH}=10$, minor small-angle scattering is observed, proving that the sample is homogeneous on a length scale from 20 to $60 \mathrm{~nm}$, and therefore, no aggregation is observed. The $\varepsilon$-PLL aqueous solution at $\mathrm{pH}=7$ shows a more significant small-angle scattering signal, likely related to aggregation phenomena. The presence of this aggregation phenomenon can explain the water crystallization observed at this low $\mathrm{pH}$ value [see Fig. 3(c)] since the aggregation should result in less hydration water and thereby more bulklike water that easily crystallizes.

Regarding the dynamics, when the $\mathrm{pH}$ value decreases from 10 to 9 , no significant differences are observed in the temperature dependences of the relaxation times. However, at lower $\mathrm{pH}$ values ( 7 and 8), water crystallizes (on both cooling and heating), and therefore, the dielectric measurements only cover the temperature interval from 120 to $200 \mathrm{~K}$. The fast water relaxation becomes faster at $\mathrm{pH}=7$ and 8 than that in the amorphous sample at $\mathrm{pH}=10$.

\section{DISCUSSION}

\section{A. The fast water relaxation (the bulklike water process)}

As mentioned in the Introduction, the faster water relaxation is universal for all aqueous solutions and confined water and therefore can be considered as a more intrinsic water relaxation than the slower one, which is only present in a limited number of solutes. Figure 4 shows comparisons with bulk liquid water (black circles) at high temperatures, BDS results obtained for low density amorphous ice (LDA, open black squares ${ }^{27}$ ), and dielectric ${ }^{28}$ and $\mathrm{NMR}^{29}$ results of water confined in MCM41. The fast water relaxation in aqueous 


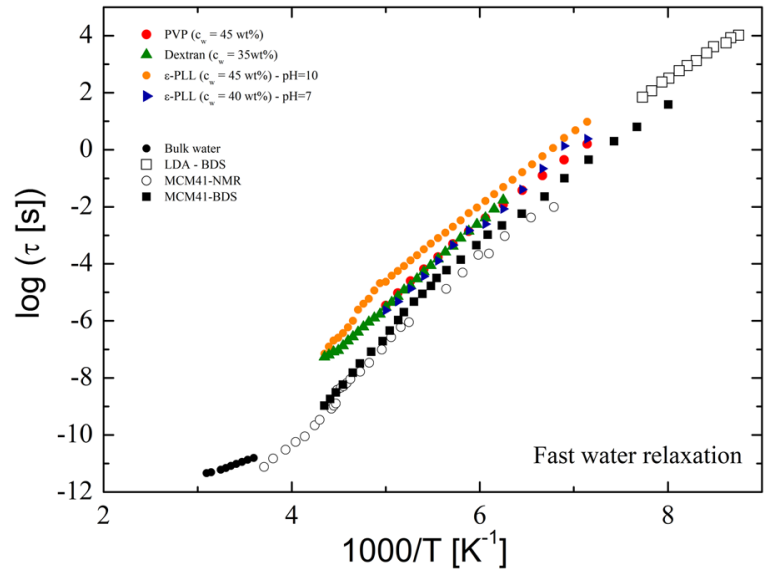

FIG. 4. The fast water relaxation in different solutions at different water contents as indicated in the figure. In addition, data of bulk water, LDA, ${ }^{27}$ and water confined in MCM41 measured by BDS ${ }^{28}$ and $\mathrm{NMR}^{29}$ are shown.

solutions of $\varepsilon$-PLL is slightly slower than bulk water and more clearly slower than water confined in MCM41, in which we can access the supercooled region without crystallization.

First, we discuss the fact that in the $\varepsilon$-PLL solution, the fast water relaxation becomes faster at low $\mathrm{pH}$ values as shown in Figs. 3(c) and 4. This should be related to the fact that the reduction in $\mathrm{pH}$ gives rise to a change of the conformation from $\beta$-sheets to a mix between $\beta$-sheets and $\beta$-turns of $\varepsilon$-PLL. However, provided that we identify this relaxation to a local bulklike water process (and therefore with little influence from interactions with the solute), it should not be directly affected by the conformation of $\varepsilon$ PLL but only indirectly due to partial water crystallization at low $\mathrm{pH}$. Although the crystalline regions of water may produce "hard confinements" for the amorphous water and thereby alter this water relaxation to relaxation times typical of water in hard confinements, it should be noted that results for both water solutions ${ }^{30}$ and water in MCM $41^{31}$ have not indicated any change in the water dynamics due to ice formation. Therefore, another possible explanation to consider is the fact that the state of protonation of carboxyl $(\mathrm{COOH})$ and amine $\left(\mathrm{NH}_{2}\right)$ groups changes at the different $\mathrm{pH}$ values. $\mathcal{E}$-PLL, at $\mathrm{pH} 7$ or 8 , has both amine and carboxyl groups protonated $\left(\mathrm{COO}^{-}\right.$and $\left.\mathrm{NH} 3+\right)$, and this could be the reason behind the different aggregation of water. This could affect the concentration of free ions in the solution and forces water to aggregate as indicated in the SAXS measurements. The change of $\mathrm{pH}$ can modify the geometrical packing of water molecules around them. In fact, early dielectric spectroscopy experiments ${ }^{32}$ on concentrated salt solutions showed that the water dynamics becomes faster when more ions are in the solution. This is likely a result of a distorted water structure and a reduction in hydrogen bonds between the water molecules.

Moreover, in Fig. 5, we show a comparison of the dynamics of water in solutions with that observed in MCM41, which is considered a model system for confined water. It is important to note that the strong concentration dependence of the relaxation time of the fast water [from times represented in Fig. 5(a) to those in Fig. 5(c), there are 4 decades] is common for aqueous solutions but in contrast to the situation for water in hard confinements, as discussed in Ref. 14. In the following, we considered three different cases: (a) water content lower than 0.5 wt. \%, (b) the highest water content before observing crystallization, and (c) systems with a dual relaxation of water molecules. At very low water content $\left[c_{w}<0.5\right.$ wt. $\%$, Fig. 5(a)], water relaxes via an Arrhenius process ${ }^{33}$ (no crossover in its relaxation time) with an activation energy of $E_{a}=0.46 \mathrm{eV}$. This relaxation was attributed to a local relaxation of water molecules because no environmental dependency can be observed for different solutes. This relaxation is 3-4 decades slower than water in MCM41 [see Fig. 5(a)]. Increasing the water content up to a maximum level before we observe crystallization (the precise water content depends on the solute), the water relaxation becomes faster and
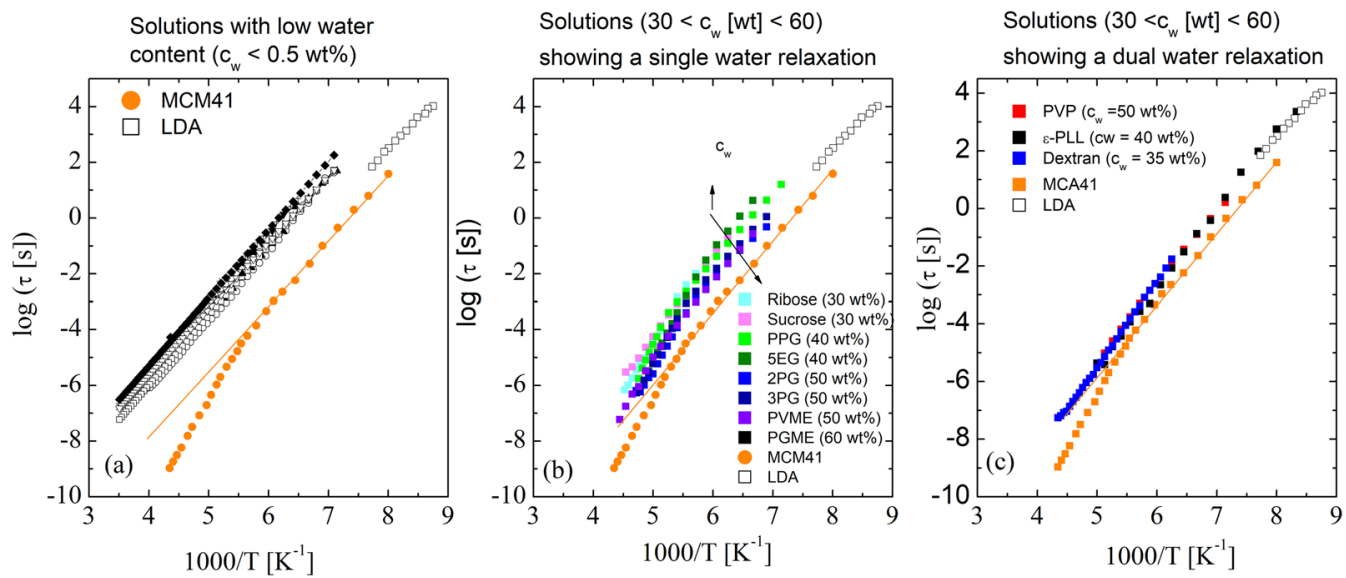

FIG. 5. Temperature dependence of the relaxation times of solutions showing a single water relaxation (a) at very low water content ${ }^{33}$ and (b) at high water content. (c) Temperature dependence of the fast water relaxation in PVP, dextran, and $\varepsilon$-PLL (i.e., solutions showing a dual water relaxation) with different water contents. Orange circles are the relaxation times of water in MCM $41,{ }^{28}$ and black squares are the relaxation times of LDA. ${ }^{2}$ 
shows a crossover at the glass transition temperature of the solution, as measured by DSC, as shown in Fig. 5(b). At low temperatures (when the relaxation time follows an Arrhenius behavior), the water molecules at the highest water content ${ }^{14,34}$ relax with an activation energy of $E_{a}=0.54 \mathrm{eV}$. However, the relaxation times are still 1.5-2 decades slower than water in MCM41, and therefore, we expect certain interactions of water with the solute molecules. If we now consider the solutions for which a dual relaxation of water is observed [Fig. 5(c)], we can see that their relaxation times are much closer to that of confined water in MCM41 (about half a decade slower). It is important to note that in this case, the water content in the samples is not an important parameter (35 wt. \% for dextran and 30-55 wt. \% for PVP) as the relaxation times of the fast relaxation are fairly independent of the water content. Therefore, comparing with the model system for supercooled water (orange filled circles) as well as with LDA for bulk water (black open squares), we can also rationalize that the fast water relaxation in these particular systems can be considered as bulklike water with little interaction with the solute molecules. This agreement with the relaxation of bulk water has important implications and will therefore be further discussed below.

\section{$B$. The relationship of the fast water relaxation with bulk water}

In Fig. 4, we compare the present results with literature data for bulk water at high and low temperatures. In Ref. 27, the dielectric relaxation of LDA, as shown in Fig. 4, was assigned to the a-relaxation of bulk water. Thus, it was believed that LDA undergoes a true glass-to-liquid transition when the temperature is raised above approximately $130 \mathrm{~K}$, in consistency with the most accepted glass transition temperature of bulk water ${ }^{35}$ at $136 \mathrm{~K}$. However, both from the confinement studies, and the universality of this water relaxation, as mentioned above, and other studies of supercooled and glassy bulk water, the assignment of the calorimetric feature at about $136 \mathrm{~K}$ to a true glass transition (in the sense that water is a viscous liquid above this temperature) has been more and more questioned in recent years.

Instead, it has been suggested ${ }^{36-39}$ that the true glass transition of bulk water is located close to $228 \mathrm{~K}$ and that the relaxation process observed below this temperature should be therefore assigned to a more local $\beta$-relaxation in a macroscopically solid material. In fact, Shephard and Salzmann ${ }^{39}$ compared the believed glass transition of LDA with the calorimetric feature observed for hydrogen disordered crystalline ice phases and found that the calorimetric feature is basically identical for the two types of systems. Furthermore, they observed that the glass-transition-like feature is not affected by isotope substitution of ${ }^{16} \mathrm{O}$ to ${ }^{18} \mathrm{O}$ but significantly shifted for $\mathrm{D}_{2} \mathrm{O}$. This implies that this calorimetric feature is due to an unfreezing of molecular reorientation dynamics, rather than due to diffusional motions of the oxygen atoms. Because of the similarity between the LDA and the hydrogen disordered crystalline ice phases, it was suggested that also LDA undergoes an unfreezing of local molecular reorientations at the believed glass transition at about ${ }^{40}$ $136 \mathrm{~K}$. This interpretation of the calorimetric feature also explains its anomalously small change in heat capacity, which has a typical value of disordered crystals, including the hydrogen-disordered ices, ${ }^{41}$ but is only $2 \%$ of the glass-to-liquid transition of, for example,
$\left(\mathrm{H}_{2} \mathrm{O}\right)_{2}-\mathrm{H}_{2} \mathrm{O}_{2}$ and $\mathrm{H}_{2} \mathrm{O}-\mathrm{N}_{2} \mathrm{H}_{4}$. If the calorimetric feature at about $136 \mathrm{~K}$ is not a true glass-to-liquid transition, it also implies that the associated dielectric relaxation process ${ }^{27}$ cannot be associated with the viscosity related structural $\alpha$-relaxation. Instead, it should, in consistency with the confinement studies, be regarded as a more local ( $\beta$-like) process. As mentioned above, the real glass-to-liquid transition is instead expected to occur at a temperature where a fragile-to-strong transition has been proposed, i.e., around $225 \mathrm{~K}$. This apparent fragile-to-strong transition would then, in reality, be a crossover from a high temperature $\alpha$-relaxation to a low temperature $\beta$-relaxation. Such a crossover is also observed for the water in solutions and hard confinements but occurs at a lower temperature in such systems due to the difficulty to form a similar rigid tetrahedral network structure as in bulk water. This geometrically restricted network structure in confinements leads to a lower dynamic crossover temperature (related to a liquid-to-glass transition), as indicated by a large number of dielectric relaxation measurements as well as fast scanning calorimetry. ${ }^{43}$ However, below the crossover temperature, it is likely that the dynamic behavior of bulk water (i.e., LDA) and confined water is very similar, as indicated in Fig. 4.

\section{Slow interfacial water dynamics-Relationship with solute dynamics}

Now, we will turn to the slow water process. If the fast water relaxation is intrinsic in character and therefore similar to bulk water, then it is important to understand why this additional water relaxation appears and what the molecular origin of it is, given that it is present only in some types of water containing systems. Below, we will try to answer this question.

As discussed in a previous publication, ${ }^{13}$ the slow relaxation of water needs a minimum number of water molecules to be observed. It is not seen at a very low hydration level $\left(c_{w}=5 \mathrm{wt}\right.$. \%) but appears at about $20 \mathrm{wt}$. \% of water. Therefore, it is likely that some kind of connectivity between the water molecules is required for observing it. Another indication for that is that the temperature dependence of its relaxation times is strongly non-Arrhenius above the glass transition temperature $T_{g}$, as typical for the cooperative a-relaxation.

In the case of proteins, they are commonly studied in welldiluted aqueous solutions, which implies that its water crystallizes on cooling down to $T_{g}$ and recrystallizes during heating. This crystallization prevents the observance of the slow water relaxation at temperatures close to $T_{g}$. The slow water relaxation for proteins was therefore only clearly observed ${ }^{44}$ in the temperature range lower than $T_{g}$ and in some cases for some degrees above $T_{g}$. However, for the nonbiological systems here analyzed, crystallization is avoided (even at normal cooling rates of $1-10 \mathrm{~K} / \mathrm{min}$ ) for the whole temperature range and therefore it is possible to detect the slow relaxation of water molecules in a very broad temperature range, as shown in Fig. 6 for such systems. The appearance of this slow water relaxation is in contrast to most aqueous solutions, where only the fast water relaxation is observed. ${ }^{14}$ Therefore, the question is, what water in these studied systems have in common with water in protein solutions? To answer this question and the previous question about its origin, it is important to make two observations. First, as shown in Fig. 6, the time scale and temperature dependence of this slow water relaxation are strongly dependent on the solute molecule, and 


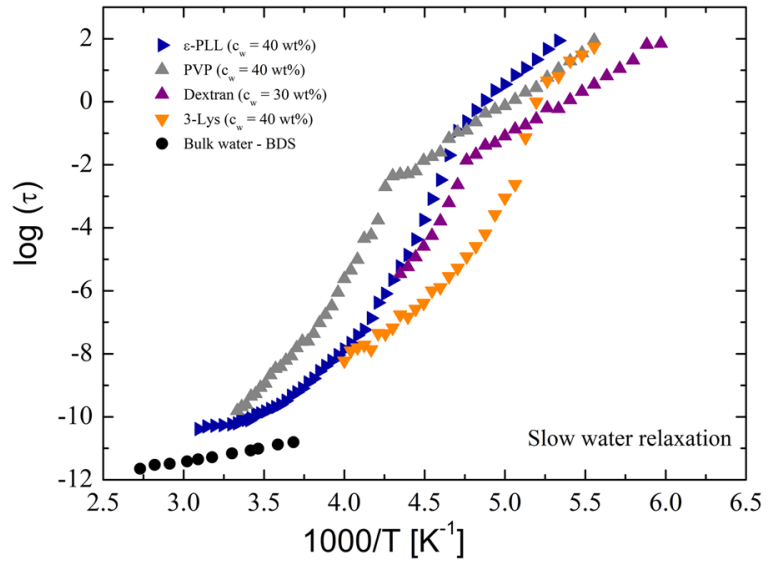

FIG. 6. Temperature dependence of the relaxation times of the slow water relaxation in different systems as indicated in the figure. Black circles represent the relaxation times of bulk water.

second, as shown in Fig. 7, the temperature dependence of this water relaxation is the same as the temperature dependence of the solute relaxation, i.e., the solute relaxation is "slaved" by this water relaxation. ${ }^{45}$ The first observation indicates that the water involved in this water relaxation is strongly influenced by the interaction with and motions of the solute molecules, i.e., this water relaxation is probably not involving only the water motions, but is more likely a collective and coupled motion of water molecules and surface groups of the solute molecules. Thus, the water participating in this relaxation process is likely directly interacting with the solute molecules. The second observation shows that this coupled water-solute relaxation is directly responsible for the slower glass transition related main relaxation of the solute molecules, which, in turn, is responsible for the major protein activity in the case of proteins. Hence, it seems as the "slaving phenomenon" is directly related to the appearance of this slow additional water (-solute) relaxation, which is not observed in other types of solutions.

The reason why this water related relaxation is not observed in most aqueous solutions is not fully clear because water-solute interactions should also occur in the other systems, but for some reason, they do not have the same coupling between water and solute motions. Most likely, the relaxation dynamics of these other solute molecules are not strongly dependent on the presence of the surrounding water (although water also in these systems generally acts as a plasticizer and speeds up the solute dynamics), and thereby, there is no direct coupling between the water and solute dynamics. Typical for the systems where a single water relaxation is observed is that the glass transition temperature of the pure solute is considerably lower than for the solutes where the slow water related relaxation is observed in their solutions. ${ }^{15}$ Thus, in the latter systems, there is an intimate relation between the water and solute dynamics, where the slow water relaxation appears as a coupled water-solute relaxation, which, in turn, is responsible for (or "slave") the glass transition related global motions of the solute molecules. Moreover, when these global solute molecules undergo a glass transition, the nature and temperature dependence of the coupled water-solute relaxation (i.e., the process we denote as the slow water relaxation) change dramatically to a low temperature Arrhenius behavior, as shown in Fig. 6. This suggests that the cooperativity of this watersolute relaxation is lost when the global solute motions freeze in. Thus, it transforms to a $\beta$-like relaxation at the glass transition of the solute. In that sense, this slow water relaxation is not only coupled to relaxations of surface groups of the solute molecules but also dependent on the global solute motions. This implies that there is a complex coupling and relation between the water and solute dynamics on different time scales, where the water dynamics plays an essential role for the solute dynamics, but where there is also a kind of "feedback mechanism" where the solute dynamics, in turn, promote the cooperative water motions. In ordinary solutions where the slow cooperative water relaxation is absent, there is no such intimate and complex relation between the water and solute dynamics. Instead, the global solute motions occur even without water, and the only role of water is to act as a plasticizer for the solute.

\section{Other interpretations related to the presence of two water relaxations}

In this section, we discuss and compare our results with other findings in the literature related to the presence of two water
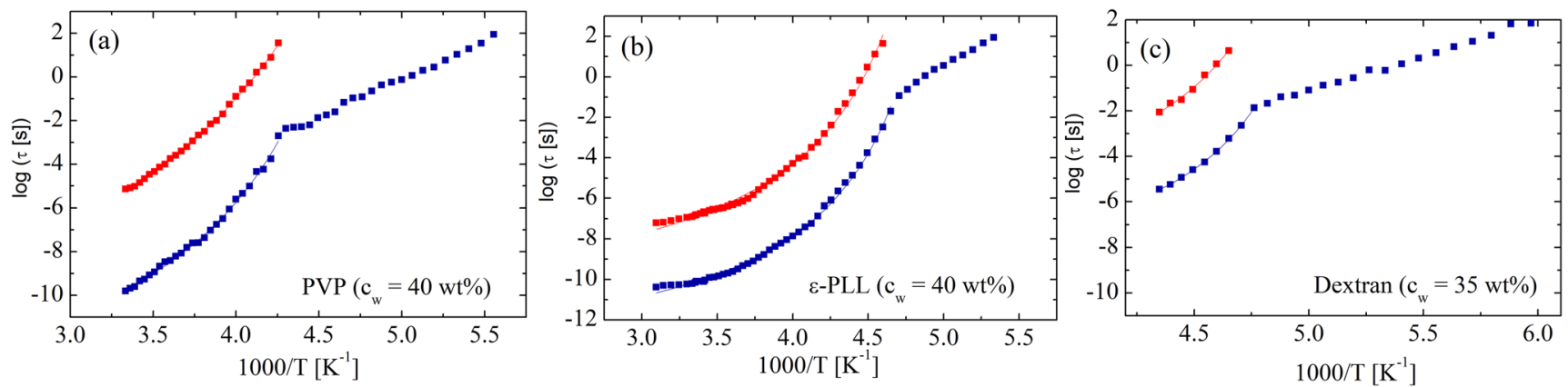

FIG. 7. Temperature dependence of the relaxation times for the systems and water contents indicated in each figure. The red squares represent the solute relaxation, whereas the blue squares represent the slow water relaxation. 
relaxations, which have been observed in water solutions or even in bulk water.

Two water relaxations have been observed in a previous study on the dynamics of bulk water by means of neutron scattering and dielectric spectroscopy at room temperature. ${ }^{46}$ The slower mode was assigned to a supramolecular process [the intense peak observed in Fig. 1(c) for bulk water and related to the so-called Debye peak also observed in mono-alcohols ${ }^{47}$ ], whereas the fast mode was related to the structural ( $\alpha$-relaxation) of bulk water. We can therefore interpret our slow and fast water relaxations as these two modes where the "slow water relaxation" could be identified with the supramolecular process of bulk water and the "fast water relaxation" to the structural (a-relaxation) of water. It is important to note that whereas the slow dielectric water process for bulk water is a Debyelike peak (i.e., single exponential), the slow relaxation discussed here is more extended and one decade slower than the Debye peak of bulk water as observed in Fig. 1(c). If all the considerations of the fast water relaxation in Sec. IV B are taken into account, we consider it unlikely that these two water relaxations in the three aqueous solutions analyzed in this work can be related with those observed in bulk water.

On the other hand, and following the results of a very recent molecular dynamics simulation, ${ }^{48}$ it was found that binary mixtures of waterlike molecules could cause microphase separation at low temperatures $(100-150 \mathrm{~K})$. In the simulation, two types of waterlike molecules were used (which differ in the partial charges of $\mathrm{H}$ and $\mathrm{O}$ atoms) and two different dynamical processes were observed, each one with different characteristics. These binary mixtures can be considered as ideal models of binary glass forming liquids with high dynamical contrast, as the solutions studied in this work. Therefore, we need to also explore the possibility that microphase separation could be the cause to find two different water relaxations. However, following the fact that the samples do not crystallize (except $\varepsilon$-PLL at low $\mathrm{pH}$ ) on cooling, we can rule out microphase separation, at least on length-scales above $3 \mathrm{~nm}$.

\section{CONCLUSIONS}

In this work, we have studied the dynamics of certain nonbiological aqueous solutions (in particular, $\varepsilon$-PLL) which resemble the dynamics of protein solutions but have the advantage that they can be studied fully dissolved in water without any crystallization. This makes it possible to study a unique slow water relaxation over a broad temperature range, where ice formation prevents its observation above the glass transition of the protein. We have discussed the nature of this unique water relaxation, which in addition to protein solutions has only been observed in a few other types of systems. Common for all these systems is that it determines (or "slaves") the global motions of the solute molecules, which would be absent or only present at very much higher temperatures, without the surrounding water. However, the analysis of this slow water relaxation indicates that also surface groups or certain structural units of the solute molecules participate in the process, which makes it to a coupled water-solute relaxation. Above the glass transition, the temperature dependence of this coupled water-solute relaxation is highly non-Arrhenius, as typical for cooperative relaxation processes involving several molecules or structural units, but below $T_{g}$, it transforms to an Arrhenius activated relaxation process of $\beta$-like character. Thus, it seems as its cooperative nature disappears when the global solute motions freeze in. This implies that the dynamical relation between the water and the solutes is more complex than that a coupled water-solute relaxation determines (or "slaves") the global solute motions since the global solute motions seem to facilitate the cooperativity of the water-solute relaxation, resulting in an intimate relation between water and solute dynamics as well as between local and global solute motions.

We have also discussed the universality of the fast water relaxation and argued that it should be considered as an intrinsic local $\beta$-relaxation if the water content is sufficiently high to produce clusters of water molecules. Further support for this assignment is its similarity to the relaxation process of LDA.

\section{SUPPLEMENTARY MATERIAL}

See supplementary material for relaxation strengths for the fast water relaxation, the slow water relaxation, the solute relaxation of $\varepsilon$-poly(lysine) solutions, and the infrared response of the dried and wet $\varepsilon$-poly(lysine).

\section{ACKNOWLEDGMENTS}

The ELKARTEK program (nG17, Grant No. KK-2017/00012), Basque Goverment (IT-1175-19), CSIC (LINKB20012), and the Swedish Research Council (No. 2015-05434) are acknowledged for financial support. The X-ray experiments were performed at BL11NCD beamline at the ALBA Synchrotron with the collaboration of ALBA staff.

\section{REFERENCES}

${ }^{1}$ S. Ebbinghaus et al., Proc. Natl. Acad. Sci. U. S. A. 104, 20749 (2007).

${ }^{2}$ L. W. Luyuan Zhang, Y.-T. Kao, W. Qiu, Y. Yang, O. Okobiah, and D. Zhong, Proc. Natl. Acad. Sci. U. S. A. 104, 18461 (2007).

${ }^{3}$ N. Q. Vinh, S. J. Allen, and K. W. Plaxco, J. Am. Chem. Soc. 133, 8942 (2011).

${ }^{4}$ L. Nilsson and B. Halle, Proc. Natl. Acad. Sci. U. S. A. 102, 13867 (2005).

${ }^{5}$ G. Camisasca et al., J. Chem. Phys. 145, 044503 (2016).

${ }^{6}$ D. Corradini et al., Sci. Rep. 3, 1218 (2013).

${ }^{7}$ D. Laage, T. Elsaesser, and J. T. Hynes, Chem. Rev. 117, 10694 (2017).

${ }^{8}$ S. C. Harvey and P. Hoekstra, J. Phys. Chem. 76, 2987 (1972).

${ }^{9}$ G. Otting, Prog. Nucl. Magn. Reson. Spectrosc. 31, 259 (1997).

${ }^{10}$ N. Shinyashiki et al., J. Phys. Chem. B 113, 14448 (2009).

${ }^{11}$ A. Panagopoulou et al., Biochim. Biophys. Acta, Proteins Proteomics 1814, 1984 (2011).

${ }^{12}$ S. A. Lusceac, M. R. Vogel, and C. R. Herbers, Biochim. Biophys. Acta, Proteins Proteomics 1804, 41 (2010).

${ }^{13}$ S. Cerveny, I. Combarro-Palacios, and J. Swenson, J. Phys. Chem. Lett. 7, 4093 (2016).

${ }^{14}$ J. Swenson and S. Cerveny, J. Phys.: Condens. Matter 27, 033102 (2015).

${ }^{15}$ I. Combarro Palacios et al., J. Chem. Phys. 150, 124902 (2019).

${ }^{16}$ P. Papadopoulos et al., J. Chem. Phys. 122, 224906 (2005).

${ }^{17}$ A. Gitsas et al., Biomacromolecules 9, 1959 (2008).

${ }^{18}$ S. Maeda, C. Chizuru Sasaki, and K. Kunimoto, in NMR Spectroscopy of Polymers: Innovative Strategies for Complex Macromolecules Vol. 1077 ACS Symposium Series, edited by H. N. Cheng, T. Asakura, and A. D. English (American Chemical Society, 2011), pp. 317-335.

${ }^{19}$ S. Jia et al., Int. J. Mol. Sci. 10, 3358 (2009).

${ }^{20}$ A. Panagopoulou et al., J. Phys. Chem. B 116, 4593 (2012).

${ }^{21}$ H. Jansson and J. Swenson, Biochim. Biophys. Acta 1804, 20 (2010). 
${ }^{22}$ S. A. Lusceac et al., J. Non-Cryst. Solids 357, 655 (2011).

${ }^{23}$ J. Kong and S. Yu, Acta Biochim. Biophys. Sin. 39, 549 (2007).

${ }^{24}$ A. Dong, P. Huang, and W. S. Caughey, Biochemistry 29, 3303 (1990).

${ }^{25}$ S. C. Shukla et al., Biochem. Eng. J. 65, 70 (2012).

${ }^{26}$ S. Maeda et al., Polym. Bull. 68, 745 (2011).

${ }^{27}$ K. Amann-Winkel et al., Proc. Natl. Acad. Sci. U. S. A. 110, 17720 (2013)

${ }^{28}$ J. Sjostrom et al., J. Chem. Phys. 128, 154503 (2008).

${ }^{29}$ M. Sattig and M. Vogel, J. Phys. Chem. Lett. 5, 174 (2014).

${ }^{30}$ S. Cerveny et al., J. Non-Cryst. Solids 356, 3037 (2010).

${ }^{31}$ M. Weigler et al., J. Phys. Chem. B 123, 2123 (2019).

${ }^{32}$ R. Buchner, G. T. Hefter, and J. Barthel, J. Chem. Soc., Faraday Trans. 90, 2475 (1994).

${ }^{33}$ S. Cerveny et al., Phys. Rev. Lett. 93, 245702 (2004).

${ }^{34}$ S. Cerveny, J. Colmenero, and A. Alegria, Eur. Phys. J.: Spec. Top. 141, 49 (2007).

${ }^{35}$ G. P. Johari, A. Hallbrucker, and E. Mayer, Nature 330, 552 (1987).
${ }^{36}$ J. Swenson and J. Teixeira, J. Chem. Phys. 132, 014508 (2010).

${ }^{37}$ S. A. McCartney and V. Sadtchenko, J. Chem. Phys. 138, 084501 (2013).

${ }^{38}$ J. Qvist, H. Schober, and B. Halle, J. Chem. Phys. 134, 144508 (2011).

${ }^{39}$ J. J. Shephard and C. G. Salzmann, J. Phys. Chem. Lett. 7, 2281 (2016).

${ }^{40}$ J. Swenson, Phys. Chem. Chem. Phys. 20, 30095 (2018).

${ }^{41}$ C. G. Salzmann et al., Phys. Chem. Chem. Phys. 13, 18468 (2011).

${ }^{42}$ C. A. Angell, Science 319, 582 (2008).

${ }^{43}$ D. Bhattacharya, C. N. Payne, and V. Sadtchenko, J. Phys. Chem. A 115, 5965 (2011).

${ }^{44}$ K. Sasaki et al., J. Phys. Chem. B 121, 265 (2017)

${ }^{45}$ H. Frauenfelder et al., Proc. Natl. Acad. Sci. U. S. A. 106, 5129 (2009).

${ }^{46}$ J. S. Hansen et al., Phys. Rev. Lett. 116, 237601 (2016).

${ }^{47}$ J. Gabriel, F. Pabst, and T. Blochowicz, J. Phys. Chem. B 121, 8847 (2017).

${ }^{48}$ N. Müller and M. Vogel, J. Chem. Phys. 150, 064502 (2019). 